“ (C) 2013 IEEE. Personal use of this material is permitted. Permission from IEEE must be obtained for all other uses, in any current or future media, including

reprinting/republishing this material for advertising or promotional purposes, creating new collective works, for resale or redistribution to servers or lists, or reuse of any copyrighted component of this work in other works." 


\title{
Experimental Study on a Smart Wheelchair System Using a Combination of Stereoscopic and Spherical Vision
}

\author{
Jordan S. Nguyen, Steven W. Su, and Hung T. Nguyen, Senior Member, IEEE
}

\begin{abstract}
This paper is concerned with the experimental study performance of a smart wheelchair system named TIM (Thought-controlled Intelligent Machine), which uses a unique camera configuration for vision. Included in this configuration are stereoscopic cameras for 3-Dimensional (3D) depth perception and mapping ahead of the wheelchair, and a spherical camera system for 360-degrees of monocular vision. The camera combination provides obstacle detection and mapping in unknown environments during real-time autonomous navigation of the wheelchair. With the integration of hands-free wheelchair control technology, designed as control methods for people with severe physical disability, the smart wheelchair system can assist the user with automated guidance during navigation. An experimental study on this system was conducted with a total of 10 participants, consisting of 8 able-bodied subjects and 2 tetraplegic (C-6 to C-7) subjects. The hands-free control technologies utilized for this testing were a head-movement controller (HMC) and a braincomputer interface (BCI). The results showed the assistance of TIM's automated guidance system had a statistically significant reduction effect $(p$-value $=\mathbf{0 . 0 0 0 5 3 3})$ on the completion times of the obstacle course presented in the experimental study, as compared to the test runs conducted without the assistance of TIM.
\end{abstract}

\section{INTRODUCTION}

$\mathrm{S}_{\mathrm{d}}^{\mathrm{E}}$ EVERE physical disabilities can be the cause of depression, significant decreases in motivation, and loss of independence for many sufferers. The ability to control mobility-assisting devices such as powered wheelchairs is important for gaining some physical independence. However, hands-free control systems in particular require significant levels of skill, attention, and judgement from the user [1]. Without adequate control over the wheelchair, the risk of accidents and collisions increases, causing damage and injury.

Smart wheelchair technology can help assist with such tasks as collision avoidance and automated guidance. This is usually achieved through the addition of sensors, computer control, and mobile robotic algorithms and capabilities. Some well-known examples of these in research include SENA [2], Rolland [3], Hephaestus [4], and Navchair [5]. However, these all require further development for reliable

This work was supported in part by Australian Research Council under Discovery Grant DP0666942 and LIEF Grant LE0668541.

Jordan S. Nguyen is with the Faculty of Engineering and Information Technology, University of Technology, Sydney, Broadway, NSW 2007, Australia (phone: +612-9514-4441; fax: +61 29514 2868; e-mail: Jordan.Nguyen@uts.edu.au).

Steven W. Su, and Hung T. Nguyen are with the Faculty of Engineering and Information Technology, University of Technology, Sydney, Broadway, NSW 2007, Australia. real-time operation with hands-free control technologies. Furthermore, they are not adequate for effective navigation in unknown environments.

As previously documented [6], the TIM smart wheelchair's vision system is modeled on equine vision to allow the stereoscopic vision to map static objects ahead of the wheelchair in real-time, whilst the spherical vision provides dynamic obstacle detection in every surrounding direction. This wheelchair is aimed at assisting people with severe physical disabilities, and is able to interface with any hands-free control technology, such as BCIs.

A real-time operational system was produced to allow users to select directions for travel via hands-free control systems, and the wheelchair carries out these commands whilst detecting and avoiding obstacles along the way, automatically doing the finer manoeuvres, and ultimately making the travel safe. An experimental study was then organized to test the system with people who are not connected to the project in any way.

Simple performance tests were described in [6], however, these were only conducted with one person connected with the project. This paper focuses on the experimental study on the real-time performance of the TIM smart wheelchair, conducted with 10 participants, whom are not connected with this project.

In Section II of this paper, a brief vision system implementation summary from [6] and excerpts of the experimental study are presented. Section III presents results and discussions of the real-time performance of the overall designed system, as found from the experimental study results. Section IV concludes this paper.

\section{METHODS}

\section{A. Stereoscopic and Spherical Vision Combination}

The stereoscopic cameras at the front of the wheelchair provide approximately $66^{\circ}$ of vision similar to the binocular vision of a horse, and the spherical vision cameras above the back of the wheelchair provide complete $360^{\circ}$ monoscopic vision, similar to the monocular vision of the horse without the posterior blind spots. This allows 3D mapping of the local environment ahead of the wheelchair and detection of obstacles posing as potential collision dangers (dynamic in particular) all around the wheelchair.

The spherical camera configuration consists of five cameras around and one on top to allow for complete $360^{\circ}$, and more than $80 \%$ of the full sphere, including everything around and above the system [7]. This is useful in this project for detecting obstacles all around the wheelchair which may obstruct movement or rotation in the corresponding directions [6]. 
An advanced real-time obstacle avoidance system was created, as previously discussed [8], and this was created using Vector Field Histogram (VFH) and Vector Polar Histogram (VPH) concepts which were improved and adapted for use with the unique camera system of the TIM smart wheelchair. This was referred to as TIM-VPH.

\section{B. Experimental Study}

This documented part of the experimental study on the performance of the TIM smart wheelchair was conducted in corridors within the University of Technology, Sydney (UTS). This was setup where each user was required to control the wheelchair using a hands-free control interface, navigate along the corridors and through an obstacle course, and avoid moving people and obstacles along the way.

A total of 10 participants, consisting of 8 able-bodied (AB) and 2 non-able-bodied (non-AB) subjects, completed the experiments on the TIM smart wheelchair, using a headmovement system (HMC) and a brain-computer interface (BCI), separately, as two methods of hands-free control. This was conducted to gather information about the wheelchair implementation, in particular the performance and safety of the system.

The AB participants consisted of 4 males and 4 females, aged from 21 to 56 , and for the Non-AB participants (C-6 to C-7), there were two males, aged 20 and 33. Across all participants the mean age \pm standard deviation (SD) was found to be $30.3 \pm 11.3$. All $\mathrm{AB}$ participants had no prior experience using wheelchairs, and both Non-AB participants are full-time wheelchair-users.

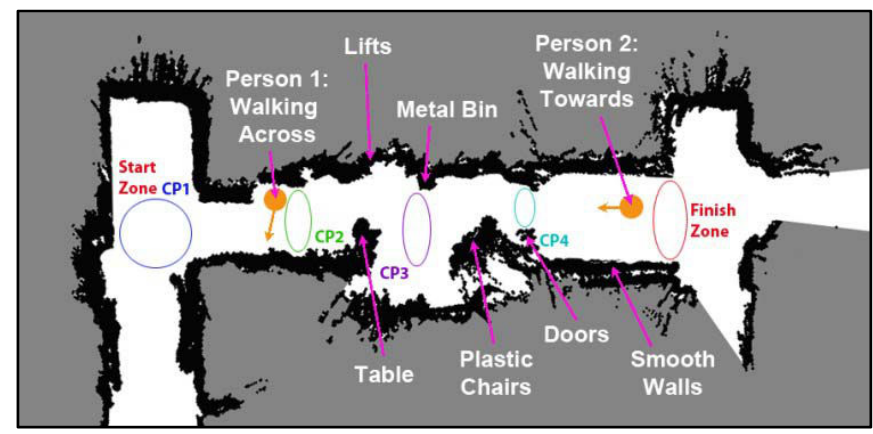

Fig. 1: 'Dynamic Course' Experimental Study Map

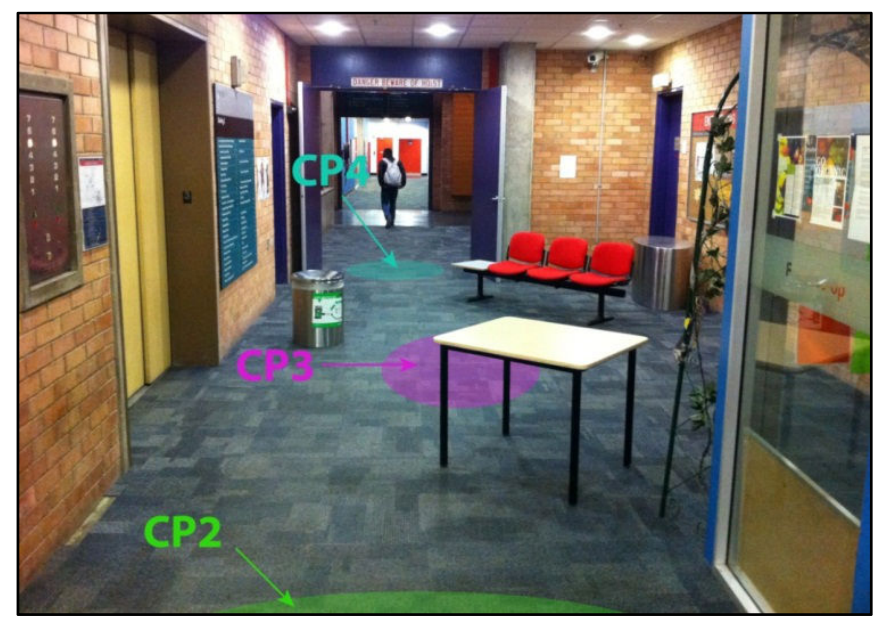

Fig. 2: Photo of part of the 'Dynamic Course'
The 'Dynamic Course' (Fig. 1), requiring roughly 29m of travel, is a test of the general static and dynamic environment navigational capabilities of the wheelchair, as well as the user's ability to control the wheelchair with the hands-free control technologies. The test is first run with a conventional joystick as a control run for comparison. Each user then completed the course with the head-movement controller (HMC) on its own with no assistance from the wheelchair (HMC Only), before being completed again with the same controller along with the automated guidance from the TIM smart wheelchair (HMC+TIM), and finally run with the brain-computer interface as the control technology with the assistance of TIM (BCI+TIM). The BCI hands-free control is not safe enough on its own so this is the reason for a BCI Only run not being conducted.

The obstacles required to be avoided in this test included tables, chairs, bins, lockers, doors, walls, general corridor features, and walking people. For this test the user was required to command the wheelchair to complete the following tasks:

- Starting at Check Point 1 (CP1), being the Start Zone in this test, and initially facing CP2 direction (Fig. 1), the user needs to command forward to the wheelchair.

- The wheelchair must automatically avoid a person walking across the path of the wheelchair in the CP2 area, before automatically passing through the CP3 obstacle course and then through the CP4 doorway.

- Immediately following the passing of the CP4 doorway the wheelchair must automatically avoid another person walking towards the wheelchair, moving in the opposite direction.

- Following this, the participant must attempt to stop the wheelchair in the Stop Zone.

These experiments test both the user's ability to control the wheelchair with the hands-free technologies, and more importantly, tests the wheelchair's ability to carry out the user's intentions, even with these new non-commercial forms of control input from the user. Of particular importance here is for the wheelchair to perceive its environment with the camera system combination in realtime and navigate safely, avoiding all obstacles.

\section{RESULTS}

Control modes analyzed in the results of the Dynamic Course experimental study are the HMC Only, HMC+TIM, and BCI+TIM, along with a joystick control mode for comparison. Fig. 3 displays results from Participant 9, a nonAB subject (C-6 to $\mathrm{C}-7$ ). The first map displays the path taken when using the HMC controller by itself without help from TIM, the second shows the HMC+TIM results and the third picture shows the BCI+TIM results.

The HMC Only run was a bit jagged in the lines of travel, with the user over-steering in the left and right directions around the path they desired to navigate. The people walking can be seen on these maps as black dots near the paths travelled. When the automated guidance of TIM was active, the people were avoided earlier and given more space than the HMC Only runs, which came close to collision at times. 


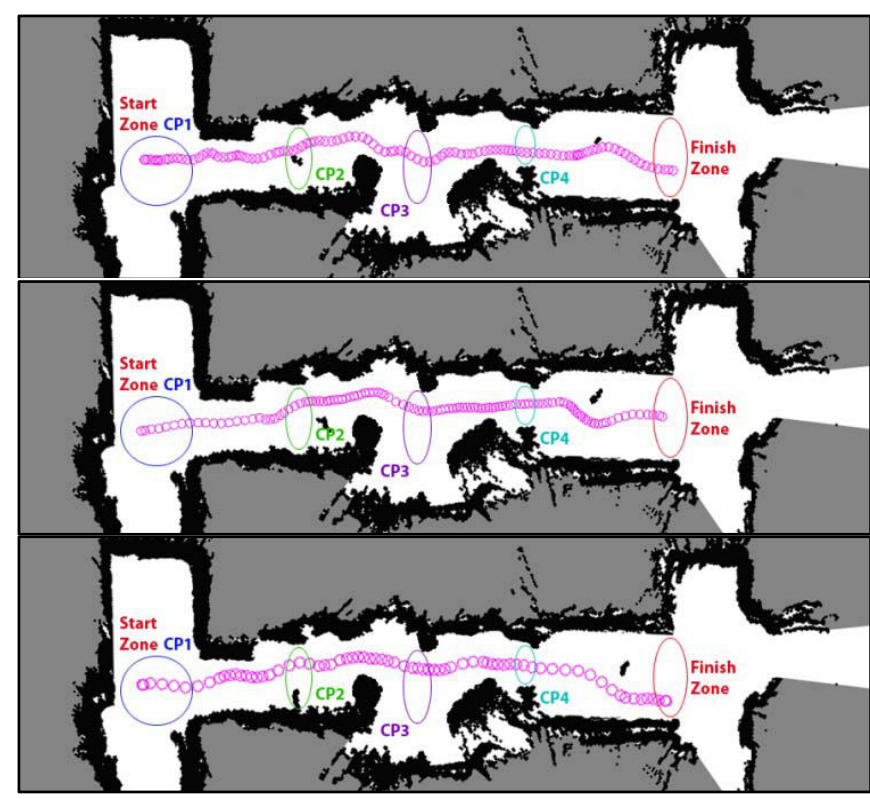

Fig. 3: Participant 9 Maps: HMC Only; HMC+TIM; BCI+TIM

Fig. 4 displays the control efforts for Particpant 9 for the paths carried out in Fig. 3. Here, the red plots the control effort for velocity and the blue plots the control effort for the steering, where the positive direction relates to the left steering direction and the negative direction relates to the right steering direction for the wheelchair.

In the first plot of Fig. 4, the HMC Only control effort displays what the participant was sending directly to the wheelchair through the head movements, and it displays a lot of oscillations with a lack of smoothness. This is viewed in contrast to the second and third plots where the HMC+TIM and BCI+TIM tests were conducted, both displaying more stability due to the majority of the steering being done by the TIM automated guidance system.

The results table data displayed in Fig. 5 shows average Dynamic Course completion times across the 10 participants, with minimum and maximum variation ranges, for each control mode. The total average test times for control modes using the automated guidance of TIM were very similar and featured low time variance between the two relevant control modes, as well as between the individual tests in each, evident in the small variation ranges of \pm 5.65 seconds and \pm 5.40 seconds, respectively. The HMC Only test took on average $13.76 \pm 0.27$ seconds longer than both of the TIM-assisted control mode tests, and also had a larger variation range of \pm 13.40 seconds. No collision interventions were necessary in any of the Dynamic Course tests, showing safe maneuvering and collision avoidance from the TIM automated guidance.

To check the significance of the time differences between when the HMC Only control mode was being used and the TIM-assisted control modes, the following test statement $(\mathrm{H})$ was produced: "In the Dynamic Course experiments, the assistance from TIM allows both HMC and BCI control methods on average across all participants to be carried out in faster course completion times than the HMC Only control".

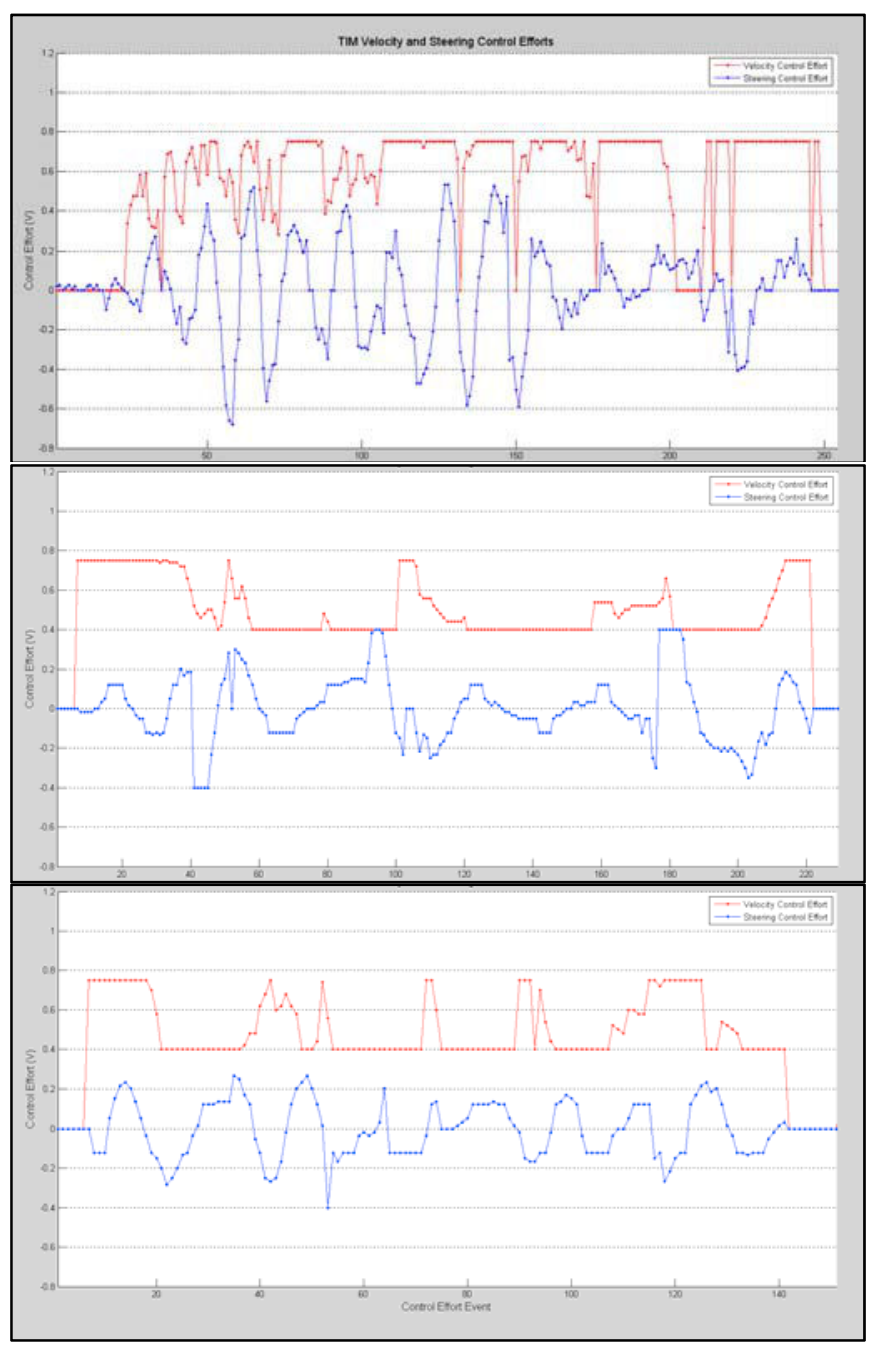

Fig. 4: Participant 9 Control Efforts: HMC Only; HMC+TIM; BCI+TIM

The HMC Only runs in the Dynamic Test produced a mean completion time of $\overline{\mathrm{x}}_{\mathrm{D}_{1}}=74.37$ seconds, with a standard deviation of $\mathrm{s}_{\mathrm{D}_{1}}=8.93$ seconds across the $n_{l}=9$ participants who completed this test. Across $n_{2}=10$ participants with the assistance of TIM, the HMC+TIM experiment runs produced a mean completion time of $\overline{\mathrm{x}}_{\mathrm{D}_{2}}=60.88$ seconds with a standard deviation of $\mathrm{s}_{\mathrm{D}_{2}}=$ 3.50 seconds, and the BCI+TIM test runs produced a mean completion time of $\overline{\mathrm{x}}_{\mathrm{D}_{3}}=60.34$ seconds with a standard deviation of $\mathrm{s}_{\mathrm{D}_{3}}=3.95$ seconds. Here, the control modes HMC+TIM/BCI+TIM data across $n_{2 / 3}=20$ participants produces a mean completion time of $\overline{\mathrm{x}}_{\mathrm{D}_{2 / 3}}=60.61$ seconds with a standard deviation of $\mathrm{s}_{\mathrm{D}_{2 / 3}}=3.47$ seconds.

One of the participants was not able to adequately move enough to use the HMC method, and hence only completed the course using the BCI control method. These small sample tests were each be analyzed using a t-distribution with a one-tailed test. For both these tests the significance level, $\alpha$, was allocated a critical value of $\alpha=0.01$, meaning if a test statement is accepted it only has a less than $1 \%$ chance of being an accident. 


\begin{tabular}{|c|c|c|c|}
\hline Participant & HMC Only & HMC+TIM & BCI+TIM \\
\hline 1 & 61.3 & 57.1 & 65.2 \\
\hline 2 & 88.1 & 54.4 & 56.0 \\
\hline 3 & 73.5 & 60.3 & 56.2 \\
\hline 4 & 85.6 & 61.6 & 60.2 \\
\hline 5 & 84.4 & 65.1 & 54.8 \\
\hline 6 & 68.2 & 65.2 & 65.4 \\
\hline 7 & 69.4 & 60.2 & 62.0 \\
\hline 8 & 72.5 & 65.7 & 60.8 \\
\hline 9 & 66.3 & 58.7 & 57.2 \\
\hline 10 & & 60.5 & 65.6 \\
\hline Mean & 74.37 & 60.88 & 60.34 \\
\hline SD & 8.93 & 3.50 & 3.95 \\
\hline Min & 61.3 & 54.4 & 54.8 \\
\hline Max & 88.1 & 65.7 & 65.6 \\
\hline
\end{tabular}

Fig. 5: Dynamic Course Completion Time Data

The degrees of freedom are:

$\left(\mathrm{n}_{1}-1\right)+\left(\mathrm{n}_{2 / 3}-1\right)=(8+19)=27$, giving a critical value of $t$, as found using a look-up table [9], as $t_{\alpha}=2.473$.

This $\mathrm{t}$-value was found to be $t=5.6365$, and therefore $t>t_{\alpha}$. This produced a p-value of $p=5.54 \times 10^{-6}$, displaying statistical significance $(p<0.001)$ in favor of the statement "In the Dynamic Course experiments, the assistance from TIM allows both HMC and BCI control methods on average across all participants to be carried out in faster course completion times than the HMC Only control".

Additionally, and in a similar fashion to this statement, testing the HMC+TIM against the HMC Only mode gave a p-value of 0.000635 and testing the BCI+TIM against the HMC Only mode gave a p-value of 0.000533 , displaying statistical significance $(p<0.001)$ in each case for significantly faster average completion times over the HMC Only mode.

\section{CONCLUSION}

This paper presents the data from the experimental study carried out on the TIM smart wheelchair, which uses a combination of stereoscopic and spherical camera systems for vision. This wheelchair is aimed at providing automated guidance assistance to people with severe physical disabilities, and this is done through its ability to interface with any hands-free control technology. The combination allows the user to send commands and the wheelchair to carry out the commands in a safe manner, automatically avoiding collisions with static and dynamic obstacles in the local environment.

An experimental study was conducted with 10 participants to test the performance of the automated guidance of TIM when interfaced to hands-free control technology. For this an obstacle course requiring about $29 \mathrm{~m}$ of navigation was setup whereby static obstacles in a corridor environment were placed as to obstruct, as well as people representing dynamic obstacles walking around. These people walked across the path of the wheelchair and another walked directly towards the wheelchair, both requiring automated collision avoidance from TIM.

The results from these experimental studies were positive, with no necessary manual collision preventions. Two handsfree control technologies, being a head movement controller (HMC) and a brain-computer interface (BCI), were trialed separately with the assistance of TIM. When compared to the use of the HMC Only, with no automated guidance assistance, it was found that the assistance of TIM allowed significantly $(p<0.001)$ faster average completion times for the dynamic obstacle course.

The results overall displayed smoothness, safety, and efficiency in navigation, when the automated guidance assistance of TIM was active. These were positive results and have been another step forward in the progress of the TIM smart wheelchair becoming an accessible and safe assistive device for people with severe physical disabilities.

\section{REFERENCES}

[1] A. Mihailidis, P. Elinas, J. Boger, and J. Hoey, “An Intelligent Powered Wheelchair to Enable Mobility of Cognitively Impaired Older Adults: An Anticollision System", IEEE Transactions on Neural Systems and Rehabilitation Engineering, vol. 15, pp. 136-143, 2007.

[2] C. Galindo, J. Gonzalez, and J. A. F-Madrigal, "Control Architecture for Human-Robot Integration: Application to a Robotic Wheelchair", IEEE Trans. on Systems, Man, and Cybernetics, pp. 1053-1067, 2006.

[3] A. Lankenau, and T. Rofer, "A versatile and safe mobility assistant", IEEE Robotics and Automation Magazine, vol. 8, pp. 29-37, 2001.

[4] R. C. Simpson, D. Poirot, F. Baxter, "The Hephaestus Smart Wheelchair System", IEEE Trans. on Neural Systems and Rehab. Eng., vol. 10, no. 2, 2002.

[5] S. P. Levine, D. A. Bell, A. J. Lincoln, R . C. Simpson, Y. Koren, J. Borenstein, "The NavChair Assistive Wheelchair Navigation System", IEEE Transactions on Rehabilitation Engineering, vol. 7, no. 4, 1999.

[6] J. S. Nguyen, Y. Tran, S. W. Su, and H. T. Nguyen, "Semiautonomous Wheelchair Developed Using a Unique Camera System Configuration Biologically Inspired by Equine Vision", 33rd Annual International Conference of the IEEE Engineering for Medicine and Biology Society, pp. 5762 - 65, 2010.

[7] J. S. Nguyen, S. W. Su, and H. T. Nguyen, "Spherical Vision Cameras in a Semi-autonomous Wheelchair System", 32nd Annual International Conference of the IEEE Engineering for Medicine and Biology Society, pp. 4064 - 4067, 2010.

[8] J. S. Nguyen, T. N. Nguyen, Y. Tran, A. Craig, S. W. Su, and H. T. Nguyen, "Real-time Performance of a Hands-free Semiautonomous Wheelchair System using a Combination of Stereoscopic and Spherical Vision", 34th Annual International Conference of the IEEE Engineering for Medicine and Biology Society, pp. 3069 - 3072, 2012.

[9] J. T. McClave and T. Sincich, Statistics: Eleventh Edition, Pearson Prentice Hall, 2009. 